\title{
LES NOUVEAUX VISAGES DE LA CONTREFACON
}

\section{LES ATTAQUES INEDITES CONTRE LES MARQUES SUR L'INTERNET : LE CAS DES MOTS-CLES DE REFERENCEMENT}

Le contrefacteur est par nature imaginatif. Sur l'internet, les opportunités de réaliser des contrefaçons sont multiples, et de manière générale, on assiste à un accroissement des attaques contre les droits de propriété intellectuelle. Si le droit d'auteur a largement monopolisé l'attention en France, la marque n'est cependant pas en reste. Les premières attaques ont porté sur l'utilisation de marques notoires à titre de noms de domaine, ainsi que sur l'usage et la reproduction des marques sur les sites. La question de la caricature et de la polémique s'est également posée à travers la construction de sites dédiés à la critique de certaines entreprises. Cependant, toute contrefaçon sur internet n'est pas nécessairement inédite, car le plus souvent la seule particularité des attaques contre les marques réside dans le support de la contrefaçon que constitue l'internet. En revanche, le mécanisme des liens tissés entre les sites afin de permettre l'interactivité de la toile multiplie les risques d'attaques contre la marque et crée de nouvelles manifestations de la contrefaçon.

Avec les liens commerciaux aussi appelés sponsorisés, ou encore promotionnels, les titulaires de sites réservent des mots-clés auprès d'outils de recherche qui procèdent alors à un référencement du site à partir des mots-clés choisis. L'internaute interroge le moteur de recherche avec des requêtes et lorsque ces dernières correspondent à des mots-clés réservés, le lien commercial sera affiché de manière privilégiée sous l'intitulé «liens commerciaux » à côté des résultats naturels générés par la requête. Le système le plus connu de référencement est Adwords de Google qui est un système de recherche payant par mots clés pour référencer les sites internet grâce aux mots faisant le plus souvent l'objet de requêtes de la part des internautes. Il est donc possible pour certains annonceurs d'utiliser la marque d'autrui à titre de lien commercial pour détourner l'internaute de l'itinéraire recherché. De nombreuses questions ont surgi avec l'utilisation des liens commerciaux, et un abondant contentieux est apparu.

S'agissant d'attaques inédites causées à la marque sur internet, la difficulté est de déterminer l'éventuelle responsabilité encourue du fait des mots-clés contrefaisants demandés par les annonceurs et fournis par les prestataires sur la demande de ces annonceurs. L'une des questions est de savoir si l'annonceur et le moteur de recherche peuvent être poursuivis de ce fait et le cas échéant sur quel fondement. Cette question a fait l'objet d'une évolution récente en droit positif. Face aux hésitations de la jurisprudence française, la Cour de justice de l'Union européenne a été saisie d'un recours préjudiciel par la Cour de cassation, dans trois affaires $^{1}$ qui mettent en cause le système Adwords de Google. Les juges français avaient tous condamné l'annonceur ainsi que Google pour contrefaçon: Google se voit reprocher de proposer des termes reproduisant des marques dans sa liste de mots clés, sans le consentement des titulaires ${ }^{2}$. Dans les trois affaires, Google a formé un pourvoi en cassation et la Cour de cassation a saisi la Cour de Justice de l'Union Européenne d'un recours préjudiciel ${ }^{3}$. Une

\footnotetext{
${ }^{1}$ Affaire Google c/ LVMH, Viaticum, Luteciel et a.) : ces affaires concernent la société Louis Vuitton Malletier SA, titulaire de la marque «Vuitton» (affaire C-236/08), la société Viaticum, titulaire des marques «Bourse des vols », «Bourse des voyages » et «BDV (affaire C-237/08), et Monsieur T., titulaire de la marque «

Eurochallenges », ainsi que la société CNRRH, licenciée de cette marque (affaire C-238/08).

${ }^{2}$ TGI Paris 4 février 2005 et CA Paris 28 juin 2006 : condamnation de Google pour contrefaçon des marques ;

TGI Nanterre 13 octobre 2003 : condamnation de Google pour actes de contrefaçon de marques. En appel, la CA de Versailles, par un arrêt du 10 mars 2005, a retenu que Google avait commis des actes de complicité de contrefaçon; TGI Nanterre 14 décembre 2004 et CA Versailles 23 mars 2006 : condamnation de Google pour contrefaçon de marque.

${ }^{3}$ Cass. com. 20 mai 2008, RLDI 2008/39, n 1292.
} 
décision fondatrice a été rendue le 23 mars $2010^{4}$ et confirmée par un arrêt du 25 mars 2010, ainsi que par un autre arrêt du 8 juillet 2010. La Cour de cassation a quant à elle rapidement fait siennes les solutions retenues par la Cour européenne par quatre arrêts de la chambre commerciale rendus le 13 juillet 2010. La Cour de cassation s'est entièrement conformée à la solution de la Cour de Justice de l'Union Européenne ${ }^{5}$. C'est donc à l'aune de ces nouvelles décisions qu'il conviendra d'étudier la question du fondement des poursuites à l'égard de l'annonceur et du prestataire de référencement, que ce soit le fondement de la contrefaçon (I) ou celui de la responsabilité civile (II).

I La qualification de contrefaçon et les mots-clés contrefaisants

Le référencement de mots-clés est une activité novatrice et la Cour de Justice de l'Union Européenne a été interpellée sur la question de l'utilisation des marques d'autrui par le biais de ce système : si la situation du client annonceur ne suscite guère de difficulté $(A)$, la position du prestataire devait être clarifiée (B).

A- La qualification de contrefaçon à l'égard du client

Il s'agit de déterminer si l'annonceur qui réserve des mots-clés correspondant à la marque d'un tiers pour désigner et vendre des produits concurrents porte atteinte au droit du titulaire de la marque. L'utilisation non autorisée de la marque d'autrui grâce au système Adwords constitue-t-elle une atteinte au droit de la marque ? Le choix de mots-clés reproduisant la marque d'autrui peut peut-être constituer un acte de contrefaçon.

En droit français, la jurisprudence sanctionne l'annonceur majoritairement sur le fondement de la contrefaçon en cas d'atteinte à une marque ${ }^{6}$, même si cette solution a parfois été remise en cause ${ }^{7}$.

Après les arrêts Google du 23 mars 2010 et Bergspechte du 25 mars 2010, la Cour de Justice s'est à nouveau prononcée sur ce point dans un arrêt Portakabin du 8 juillet 2010 dans lequel la Cour précise les conditions de cette action en contrefaçon ${ }^{8}$. La Cour de cassation a repris à son compte la solution, et la situation de l'annonceur est désormais fixée.

La contrefaçon est caractérisée lorsque l'usage non autorisé d'un signe identique à la marque a lieu dans la vie des affaires, que cet usage est fait pour des produits ou des services identiques à ceux pour lesquels la marque est enregistrée, et enfin que cet usage porte atteinte

\footnotetext{
${ }^{4}$ A propos de l'arrêt de la CJUE 23 mars 2010 : G. BONET, «Publicité sur internet et référencement selon la Cour de justice : contrefaçon de marque ou directive n ${ }^{\circ}$ 2000/31/CE ? », CCE 2010, étude 12 ; C. CASTETS-RENARD, «Système Adwords : Google n'est ni contrefacteur ni complice d'actes de contrefaçon », RLDI 2010/60, comm. n 1999 ; RLDI 2010/59, p. 3, obs. L. COSTES ; A. FOLLIART-MONGUIRAL, « CJUE, arrêt Google : droit des marques et internet : la responsabilité du prestataire de service de référencement », Propr. industr. juin 2010, comm. 38, et «CJUE, arrêt BergSpechte, droit des marques et internet : la responsabilité de l'annonceur », Propr. industr. juin 2010, comm. 39 ; L. IDOT, « Marque et référencement sur Internet », Europe 2010, comm. 181 ; J. LARRIEU, «Les liens sponsorisés rattrapés par la responsabilité civile », Propr. Industr. juin 2010, comm. 45 ; M. MALAURIE-VIGNAL, « Google : problèmes posés par le référencement de mots-clés », CCC 2010, comm. 132 ; C. MANARA, obs. D. 2010, p. 885 ; L. MARINO, « Google au pays des publicités : du droit des marques au droit de la responsabilité », JCP G 2010, note 642 ; PH. STOFFEL-MUNCK, « La notion d'hébergeur à la lumière de l'affaire Google Adwords », CCE 2010, comm. 88 ; E.TARDIEU-GUIGUES, RLDI 2010/62, comm. n 2029.

${ }^{5}$ C. CARON, « «FAQ» $\mathrm{n}^{\circ} 2$ autour des liens commerciaux », JCP ent. 2010, comm. 1961 ; M. MALAURIEVIGNAL, «Contrefaçon et utilisation de mots-clés par un moteur de recherche », CCC octobre 2010, comm. 230 ; M. SCHAFFNER, «Adwords : la clé du succès du système de mots-clés », RLDI 2010, comm. n 64 ; P. TREFIGNYGOY, «Les liens commerciaux, suite ... ou la Cour de cassation, après la CJUE », Propr. Industr. octobre 2010, comm. 66.

${ }^{6}$ CA Paris 14 mars 2001, CCE 2002, n 3, note C. CARON.

${ }^{7}$ Pour une exclusion très contestable de la contrefaçon à l'égard de l'annonceur : TGI Strasbourg 20 juillet 2007, RLDI janvier 2008, $\mathrm{n}^{\circ} 34, \mathrm{n}^{\circ}$ 1128, note E. TARDIEU-GUIGUES ; P. TREFIGNY, Propr. industr. novembre 2007, comm. 87.

${ }^{8}$ C. CARON, « «FAQ » $\mathrm{n}^{\circ} 2$ autour des liens commerciaux », précité.
} 
ou est susceptible de porter atteinte aux fonctions de la marque. Ces trois conditions sont cumulatives 9 .

L'usage par l'annonceur a bien lieu dans la vie des affaires, car il s'agit de constater que l'usage est fait dans le cadre d'une activité commerciale visant un avantage économique. L'affichage du mot-clé vise bien à conduire l'internaute sur le site marchand de l'annonceur, et l'usage était fait pour des biens identiques. Restait à vérifier la condition de l'atteinte aux fonctions de la marque, la Cour de Justice ayant précisé dans les arrêts Bergspechte ${ }^{10}$ et Portakabin que l'atteinte à la fonction de publicité devait être écartée : seule l'atteinte à la fonction d'identification de l'origine des produits et des services doit être examinée. Ce type d'atteinte variera selon la présentation de l'annonce ${ }^{11}$ : il s'agit de déterminer si l'internaute moyen peut savoir que les produits ou services proposés par le biais du lien généré par le motclé ne proviennent pas du titulaire de la marque et sont sans lien avec ce titulaire. En l'absence de confusion pour l'internaute normalement informé et raisonnablement attentif, la contrefaçon ne sera pas caractérisée faute d'atteinte aux fonctions de la marque. La qualification de contrefaçon dépendra donc de la manière dont l'annonce a été présentée. Selon la Cour de justice, il incombe à la juridiction nationale d'apprécier, au cas par cas, si les faits du litige caractérisent une atteinte ou un risque d'atteinte aux fonctions de la marque. Ainsi, l'usage de la marque d'autrui ne sera pas systématiquement sanctionné et pourrait être justifié par des motifs légitimes, comme par exemple l'usage aux fins de publicité comparative.

Au regard des évolutions récentes du droit positif, la reproduction par un mot-clé de la marque d'autrui sans autorisation pour désigner des produits ou des services identiques caractérise la contrefaçon dès qu'il existe une confusion ou un doute sur l'origine des biens commercialisés. La Cour de justice a en outre précisé dans l'arrêt Portakabin que la reproduction de la marque de manière quasi-identique, à une lettre près, relève bien de la contrefaçon par reproduction et qu'il n'est donc pas nécessaire d'établir d'existence d'un risque de confusion. La Cour de cassation a suivi scrupuleusement les solutions de la Cour de justice de l'Union Européenne. La qualification de contrefaçon est donc encourue par l'annonceur, et il fallait trancher si cette contrefaçon pouvait être retenue également à l'encontre du moteur de recherche.

B- L'exclusion définitive de la contrefaçon pour les prestataires de référencement A la suite de la situation de l'annonceur, il fallait déterminer si la contrefaçon de marques pouvait être retenue à l'égard des moteurs de recherche du fait des mots-clés contrefaisants fournis à leurs clients ${ }^{12}$.

En droit interne ${ }^{13}$, les décisions ont souvent condamné les moteurs de recherche. Cette jurisprudence est critiquable car elle retient en majorité la qualification de contrefaçon à l'encontre du moteur de recherche. La justification de la contrefaçon est en effet difficile à

\footnotetext{
${ }^{9}$ Article 5, parag.1, a), de la directive 89/104.

${ }^{10}$ Dans cet arrêt du 25 mars 2010, la Cour considère que l'utilisation de la marque comme mot-clé ne constitue pas une atteinte à la fonction de publicité de la marque : A. FOLLIART-MONGUIRAL, «Droit des marques et internet : la responsabilité de l'annonceur », précité.

11 Arrêt Portakabin point 34.

${ }^{12}$ Qualification également fondée sur les articles 5, paragraphe 1, sous a) et b) de la directive 89/104/CEE et 9, paragraphe 1, sous a) et b) du Règlement (CE) n 40/94 du Conseil, du 20 décembre 1993 sur la marque communautaire.

${ }^{13}$ Voir contra, TGI Nice 7 février 2006, RLDI 2006, $\mathrm{n}^{\circ} 14, \mathrm{n}^{\circ} 410$ : les liens publicitaires n'étaient pas de nature à induire en erreur ni à créer un risque de confusion, car «l'usage des liens hypertextes est consubstantiel à Internet, de sorte que les internautes ne sauraient se méprendre par principe sur leur utilisation». Cette analyse a été qualifiée de «très optimiste » par E. DREYER : «Un an de droit de la publicité », CCE juillet 2006, n 7, p. 7.
} 
admettre en l'absence de rapport de concurrence entre le moteur de recherche et le titulaire de la marque, et l'on voit mal en quoi le principe de spécialité serait dans cette hypothèse applicable $^{14}$. L'usage des marques dans les mots-clés par Google n'est pas réalisé pour des produits ou des services identiques ou similaires à ceux pour lesquels les marques ont été enregistrées. Ce ne serait le cas que lorsque les marques sont réservées pour des activités publicitaires ou de communication en ligne. Les juges passent souvent rapidement sur cette question pourtant cruciale ${ }^{15}$, ou peinent à convaincre ${ }^{16}$. La qualification de contrefaçon est inadéquate à l'égard du fournisseur de liens ${ }^{17}$, et ces solutions ne sont pas satisfaisantes ${ }^{18}$. Aussi doit-on se féliciter que certaines décisions condamnent le fournisseur de liens sur un fondement différent de la contrefaçon ${ }^{19}$, et notamment sur le fondement de la responsabilité civile délictuelle de droit commun ${ }^{20}$, et sur le fondement du droit spécial en cas d'usage injustifiée d'une marque notoire ${ }^{21}$.

La jurisprudence est en outre critiquable en ce qu'elle impose ainsi une responsabilité automatique du moteur du fait de la réservation des liens commerciaux litigieux par le client $^{22}$. La justification de cette position stricte consiste à considérer que le moteur dispose d'une alternative qui consiste à assumer l'intégralité des conséquences de son activité ou à y renoncer ${ }^{23}$. Ainsi, la jurisprudence a évolué vers plus de sévérité en mettant à la charge des fournisseurs des obligations alourdies : le moteur devient responsable de plein droit du fait de l'annonceur, et il ne pourra arguer d'aucun élément pour écarter sa responsabilité ${ }^{24}$.

Face aux tâtonnements de la jurisprudence, la situation des fournisseurs de liens commerciaux apparaît sous un jour plus favorable depuis la décision Google de la Cour de Justice de l'Union Européenne du 23 mars 2010. Selon la Cour, le prestataire qui offre de stocker en tant que mot-clé un terme identique à une marque, notoire ou pas, et qui organise l'affichage d'annonces à partir de celui-ci ne fait pas usage de ce signe en tant que marque dans la vie des affaires. La solution peut paraître surprenante : Google fait un usage de la marque dans la vie des affaires, puisque le référencement constitue une activité commerciale, et à ce titre est bien réalisée dans la vie des affaires en contrepartie d'un avantage économique ${ }^{25}$. Cependant, dans son arrêt du 23 mars 2010, la Cour conçoit cette notion de vie des affaires de manière très

\footnotetext{
${ }^{14}$ Pour une condamnation écartant le fondement de la contrefaçon et retenant le fondement des articles 1382 et 1383 du Code civil : TGI Paris 8 décembre 2005, précité.

${ }^{15}$ CA Versailles 10 mars 2005, précité.

${ }^{16}$ TGI Nanterre 14 décembre 2004, Propr. industr. avril 2005, comm. 26, note P. TREFIGNY.

${ }^{17}$ Considérant que la contrefaçon était applicable mais que les juges lui «ont préféré la concurrence déloyale »: P. TREFIGNY, «Les liens commerciaux : et si c'était la solution ? Quoique ... », note sous TGI Strasbourg 20 juillet 2007, précité.

${ }^{18}$ Voir encore les hésitations quant à la qualification de l'acte de contrefaçon : pour une incitation à l'usage de la marque d'un tiers : TGI Paris 24 juin 2005, RLDI 2005, $\mathrm{n}^{\circ} 10, \mathrm{n}^{\circ} 283$; ou la reproduction de la marque et l'usage de la marque reproduite : CA Aix-en-Provence 6 décembre 2007, RLDI janvier 2008, nº 1137, obs. J-B. AUROUX.

${ }^{19}$ Excluant la contrefaçon : TGI Paris 12 juillet 2006, Propr. industr. octobre 2006, $\mathrm{n}^{\circ} 10$, comm. 73, P. TREFIGNY, «Un certain pragmatisme : les mots-clés sous surveillance ».

${ }^{20}$ Pour une condamnation de Google sur le fondement de la concurrence déloyale en tant que coresponsable avec l'annonceur : TC Paris 24 novembre 2006, RLDI 2007, $\mathrm{n}^{\circ} 25, \mathrm{n}^{\circ} 803$, E. TARDIEU-GUIGUES, « Liens commerciaux : condamnation de Google en dehors du droit des marques », et $\mathrm{n}^{\circ} 24, \mathrm{n}^{\circ} 776$, obs. J-B. AUROUX. ${ }^{21}$ C'est l'article L. 713-5 du Code de la propriété intellectuelle qui permet dans ce cas de condamner le fournisseur de liens. Voir TGI Paris 12 décembre 2007, RLDI janvier 2008, n 1136.

${ }^{22}$ TGI Nanterre 14 décembre 2004, Propr. industr. avril 2005, comm. 26, note P. TREFIGNY.

${ }^{23} \mathrm{La}$ formulation de ces décisions est extrêmement sévère à l'encontre des fournisseurs de liens commerciaux, voir : CA Aix-en-Provence 6 décembre 2007, précité ; CA Aix-en-Provence 18 janvier 2008, juriscom.net.

${ }^{24}$ Selon les juges, une «impossibilité matérielle, juridique et économique » ne peut exonérer le fournisseur de sa responsabilité : CA Aix 6 décembre 2007, précité.

${ }^{25}$ Voir la décision du TPIUE ne visait que le «contexte d'une activité commerciale visant à un avantage économique »: TPIUE 10 mai 2006, JCP ent. 2006, 2747, $\mathrm{n}^{\circ} 19$, note C. CARON.
} 
stricte. Selon la Cour, «le prestataire exerce une activité commerciale et vise un avantage économique, lorsqu'il stocke, pour le compte de certains de ses clients, des signes identiques à des marques en tant que mots clés et organise l'affichage d'annonces à partir de ceux-ci. Cependant, s'il permet à ses clients de faire usage de signes identiques ou similaires à des marques, il n'en fait lui-même pas l'usage au sens des articles 5 de la directive 89/104 et 9 du règlement $n^{\circ} 40 / 94 \gg{ }^{26}$. Par conséquent, s'il est certain que le moteur de recherche, par le référencement créé, fait bien un usage de la marque d'autrui, ce n'est pas pour servir sa propre communication commerciale et pour désigner ses produits ou services : l'usage serait fait à titre documentaire ou d'indexation pour les besoins du référencement ${ }^{27}$. A défaut d'usage de la marque dans la vie des affaires, le prestataire d'un service de référencement sur internet ne peut pas être poursuivi au titre de la contrefaçon par le titulaire de la marque du fait des motsclés contrefaisants réservés par les clients. Si la contrefaçon ne devait pas selon nous prospérer à l'encontre de Google à défaut de principe de spécialité, la nouvelle conception de la notion de vie des affaires mise en exergue par la Cour n'est pas convaincante. Le moteur de recherche bénéficie aussi de l'usage de la marque d'autrui du fait de l'activité de référencement ${ }^{28}$, même s'il n'utilise pas stricto sensu les marques pour promouvoir ses propres biens.

La décision de la Cour de Justice de l'Union Européenne est donc contraire à la jurisprudence interne majoritaire qui condamne dans ce cas pour contrefaçon de marque, comme ce fut le cas devant les juridictions du fond dans les espèces concernées par le recours préjudiciel. En statuant ainsi, la Cour de Justice de l'Union Européenne donne une bénédiction quasiabsolue au système de référencement Adwords de Google, suivie en cela par la Cour de cassation qui a fait une stricte application de la solution de l'arrêt Google dans les quatre arrêts rendus par la chambre commerciale le 13 juillet 2010 : tout usage de la marque dans la vie des affaires est écarté à l'égard du prestataire de référencement. La contrefaçon ne peut être encourue désormais que par les seuls annonceurs et elle est clairement exclue à l'égard des prestataires de référencement. Il reste alors à déterminer si leur responsabilité délictuelle peut néanmoins être engagée.

II Le régime applicable au prestataire de référencement : une quasi-irresponsabilité L'exclusion de la contrefaçon conduit à s'interroger sur la responsabilité de Google à l'égard des titulaires de marque pour avoir permis à des tiers non autorisés de réserver des mots-clés correspondant aux marques. Si le régime spécial de responsabilité des hébergeurs paraît applicable à un prestataire de service de référencement (A), le retour au droit commun de la responsabilité civile est néanmoins envisageable (B).

A- La large ouverture du régime de responsabilité des hébergeurs

La Cour de cassation avait interrogé la Cour de justice de l'Union européenne pour savoir si le régime de responsabilité des hébergeurs était applicable au prestataire de référencement. Le prestataire d'un service de référencement payant peut-il être considéré comme fournissant un service de la société de l'information au sens de l'article 14 de la Directive 2000/31 du 8 juin 2000 sur le commerce électronique ? Dans ce cas, sa responsabilité ne pourrait être engagée que si, informé par le titulaire de marque de l'usage illicite du signe par l'annonceur, il n'aurait pas agi promptement pour retirer le mot-clé ou rendre l'accès à celui-ci impossible.

\footnotetext{
${ }^{26}$ Points 55 et 56 de l'arrêt.

${ }^{27}$ Ainsi est défendue l'idée que l'usage fait par Google n'emprunte pas à la fonction distinctive de la marque protégée ni à son éventuelle valeur économique ou renommée : A. FOLLIART-MONGUIRAL, « Arrêt Google : droit des marques et internet : la responsabilité du prestataire de référencement », précité.

${ }^{28}$ Sur les critiques concernant l'interprétation de la notion d'usage de la marque : C. CASTETS-RENARD,

« Système Adwords : Google n'est ni contrefacteur ni complice d'actes de contrefaçon », précité.
} 
La qualification d'hébergeur permettrait alors au prestataire de bénéficier d'un régime de responsabilité très favorable. En effet, la responsabilité de l'hébergeur ne sera engagée que si sont établis la connaissance effective par l'hébergeur de l'activité ou de l'information illicite et en outre, le fait que l'hébergeur n'a pas agi promptement pour retirer les informations ou rendre l'accès à celles-ci impossible.

Les conditions d'application de cette responsabilité ont été vérifiées par la Cour qui en a déduit l'applicabilité de ce régime sous réserve que le prestataire assure un rôle passif lors du référencement. En effet, la Cour de Justice de l'Union Européenne admet que l'entreprise Google fournit un service de la société de l'information et assure une activité d'hébergement. Par ailleurs, la Cour affirme l'indifférence de la rémunération reçue par le prestataire : ainsi, la qualification d'hébergeur n'est pas remise en cause par le fait que le prestataire soit rémunéré pour l'usage de ces signes par ses clients ${ }^{29}$. Par conséquent, la perception de rémunération et la détermination des modalités de paiement n'interfèrent pas en principe dans la mise en œuvre du régime de responsabilité des hébergeurs.

En revanche, la Cour rappelle l'exigence de passivité pour bénéficier du régime des hébergeurs. En effet, l'activité du prestataire de services doit présenter un caractère " purement technique, automatique et passif », impliquant que le prestataire «n'a pas joué un rôle actif de nature à lui confier une connaissance ou un contrôle des données stockées ${ }^{30}$.

Dans la décision Google du 23 mars 2010, la Cour de Justice renvoie à l'appréciation des juges nationaux après avoir précisé que le régime de responsabilité des hébergeurs peut s'appliquer aux prestataires de référencement. Il s'agit en fait de déterminer si le prestataire a effectivement une connaissance ou un contrôle des données stockées, à savoir des mots-clés contrefaisants réservés. La résolution du contentieux suppose de trancher si le prestataire de référencement influence l'annonceur dans le choix des mots-clés et dans la rédaction de son message accompagnant le lien. Il devra également analyser le rôle de Google dans les modalités de fixation de sa rémunération.

Le rôle des prestataires de référencement a fait l'objet d'un renvoi de la Cour de justice à l'appréciation par la juridiction nationale. Cependant, la Cour de cassation n'a pas précisé la teneur du rôle du prestataire car elle a cassé les arrêts d'appel en leur reprochant de ne pas avoir examiné l'existence de ce rôle actif. L'évolution récente paraît toutefois favorable aux prestataires de référencement, et la reconnaissance d'un rôle actif semble peu probable. La responsabilité spécifique des hébergeurs semble donc devoir régir le contentieux du référencement à l'égard des prestataires, et seule la reconnaissance de leur rôle actif permettrait de recourir à la responsabilité civile de droit commun.

B- La responsabilité de droit commun du prestataire de référencement

Parce que la Cour de justice n'a pas imposé à titre principal le régime des hébergeurs aux prestataires de référencement, il est possible de leur appliquer le régime de responsabilité du droit commun. Le rôle actif du prestataire de référencement écarterait le régime de responsabilité des hébergeurs, et les articles 1382 et 1383 du Code civil pourraient trouver application pour engager la responsabilité de Google du fait de son système Adwords. La responsabilité délictuelle du prestataire pourrait être retenue sur plusieurs fondements, tels que par exemple la concurrence déloyale ou le parasitisme ${ }^{31}$. En outre, d'autres incriminations sont envisageables, telle celle de la pratique commerciale trompeuse.

\footnotetext{
${ }^{29}$ Point 57 de l'arrêt.

${ }^{30}$ Points 113 et 120 .

${ }^{31}$ Sur les divers fondements d'action possibles, voir J. LARRIEU, « Les liens sponsorisés rattrapés par la responsabilité civile », Propr. industr. juin 2010, comm. ${ }^{\circ} 45$.
} 
En effet, le doute est permis concernant le critère de passivité, car l'activité du système Adword ressemble plus à une régie publicitaire ${ }^{32}$, ce qui lui interdirait la voie du régime des hébergeurs.

Pour démontrer sa neutralité dans l'activité de référencement, Google a adopté une nouvelle stratégie. Cette dernière est supposée ôter toute ambiguïté concernant le rôle passif de Google : Google ne contrôle plus le dépôt des mots-clés par les annonceurs depuis le 14 septembre 2010, date de la mise en place d'une nouvelle procédure Adwords ${ }^{33}$. Il s'agit d'éviter le reproche de connaissance ou de contrôle sur les données stockées et en conséquence le caractère actif du prestataire. Par ailleurs, une nouvelle procédure de retrait des annonces a été exposée par Google. Il s'agit pour les titulaires de marques d'adresser à Google une plainte relative à une annonce utilisant leur marque de sorte qu'il puisse exister pour l'internaute une confusion quant à l'origine des produits ou des services des entreprises. Le pouvoir de contrôle et de filtrage ainsi octroyé au prestataire paraît immense et flou : il suscite de nombreuses interrogations ${ }^{34}$ et pourrait aboutir à la solution contraire à celle recherchée par Google. De tels moyens de contrôle peuvent amener les prestataires à avoir une connaissance réelle des contenus surveillés, puisque la procédure appelle à une intervention spécifique du prestataire. En effet, on pourrait considérer que le prestataire joue un rôle actif de nature à lui conférer une connaissance ou un contrôle des données stockées. A trop se reconnaître de pouvoirs, Google peut modifier la nature de son rôle et être reconnu comme un intermédiaire actif du fait de la maitrise des annonces publiés dans le système Adwords.

La contrefaçon de marque retenue au titre du référencement sur internet disparaît à l'égard des prestataires : si la solution apparaît orthodoxe d'un point de vue juridique, le régime des hébergeurs proposé en substitution ne paraît ni convaincant ni juste. Cette solution aboutit à une quasi-irresponsabilité de fait des prestataires de référencement, alors même que la gestion du système de référencement paraît antinomique avec l'idée d'un rôle passif du prestataire. Ce déséquilibre pourrait être corrigé par l'application du droit commun de la responsabilité civile afin de ne pas laisser les titulaires de droit dépourvus de tout moyen d'action tout en préservant le modèle économique des moteurs de recherche.

Alexandra Mendoza-Caminade Maître de conférences- HDR Université de Toulouse 1 Capitole Faculté de droit- Centre Droit des Affaires Directrice M2 Propriété intellectuelle

\footnotetext{
${ }^{32}$ Voir notamment en ce sens, L. MARINO, précité.

${ }^{33}$ M. SCHAFFNER, « Adwords : la clé du succès du système de mots-clés », RLDI 2010, 64.

${ }^{34}$ M. SCHAFFNER, précité.
} 